\title{
Upper limb fractures in rugby in Huddersfield 1986-1990
}

\author{
K.S. Eyres FRCS ${ }^{1}$, A. Abdel-Salam FRCS ${ }^{2}$ and J. Cleary FRCS ${ }^{3}$ \\ ${ }^{1}$ Research Registrar, Department of Human Metabolism and Clinical Biochemistry, University of Sheffield, \\ Sheffield, UK \\ 2 Orthopaedic Registrar, Huddersfield Royal Infirmary, Huddersfield, UK \\ ${ }^{3}$ Orthopaedic Consultant, Huddersfield Royal Infirmary, Huddersfield, UK
}

\begin{abstract}
Most injuries sustained by rugby players affect the soft tissues, and fracture is relatively uncommon. Whereas the lower limb is most affected in footballers, the upper limb tends to be injured in rugby players. Thirty consecutive fractures and ten dislocations affecting the upper limb, sustained by 35 rugby players, are reported.
\end{abstract}

Keywords: Rugby injury, hamate fracture, sports injury

Most injuries sustained in rugby matches are to the soft tissues. Fractures are relatively uncommon, estimated to account for only $4 \%$ of injuries in adult matches and for $5 \%$ of injuries in school matches ${ }^{1}$. Whereas lower limb fractures are more common in footballers, the upper limb is more often injured in rugby players ${ }^{2}$. The upper limb injuries sustained by 35 amateur rugby players are presented.

\section{Patients and injuries}

From 1986 to 1990, 25 patients presented with 30 fractures and ten patients presented with ten dislocations of the upper limb sustained during rugby matches. All patients were male, of mean age 26 years (range 13 to 45 ). Single fractures occurred in 22 patients, two fractures occurred in two patients and one patient sustained four fractures. The distribution of the 30 fractures is shown in Table 1. The distribution of the dislocations is shown in Table 2.

Eight $(27 \%)$ of the fractures were caused by a fall while running with the ball, $18(60 \%)$ fractures occurred either when tackling or being tackled and four $(13 \%)$ fractures occurred in the scrummage. Most of the injuries (73\%) occurred in players in the wing- or prop-forward positions. The three patients with multiple fractures are described below.

Address for correspondence: K.S. Eyres, Department of Human Metabolism and Clinical Chemistry, Beech Hill Road, University of Sheffield, Sheffield S10 2RX, UK

(C) 1991 Butterworth-Heinemann Ltd.

0306-3674/91/030139-03

\section{Case 1}

A 24-year-old full-back fractured his left clavicle in a scrummage in 1988 (Figure 1). Six months later, he sustained a direct blow to the left shoulder. Radiographs showed a fracture to the greater tuberosity of the left humerus (Figure 2). He was treated with a collar and cuff and was able to return to matchplay after 2 months. Nine months later he fell onto his left hand after a tackle. Radiographs showed a sagittal fracture of the body of the hamate and a fracture of the base of the fourth metacarpal (Figure 3). He was treated conservatively with plaster immobilization, and after 6 weeks had regained a full range of painless wrist and finger movements. There was no evidence of coexistent metabolic bone disease.

Table 1. The distribution of the 30 fractures

\begin{tabular}{lr}
\hline Site & No. \\
\hline Clavicle & 4 \\
Humerus: tuberosity & 1 \\
Radius/ulnar: mid-shaft & 5 \\
Radial head & 3 \\
Distal radius & 5 \\
Scaphoid & 2 \\
Hamate & 2 \\
Bennett's fracture & 3 \\
Metacarpal fracture & 5 \\
Total & 30 \\
\hline
\end{tabular}

Table 2. The distribution of upper limb dislocations

\begin{tabular}{lr}
\hline Joint & No. \\
\hline Acromioclavicular joint & 3 \\
Glenohumeral joint & 5 \\
Proximal interphalangeal joint & 2 \\
Total & 10
\end{tabular}




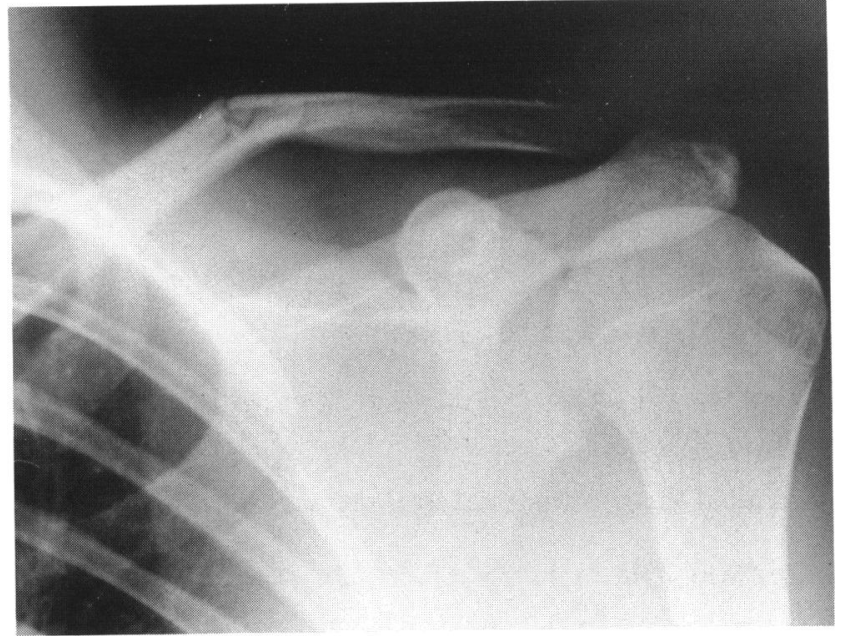

Figure 1. Case 1, first fracture - of the outer third of the left clavicle

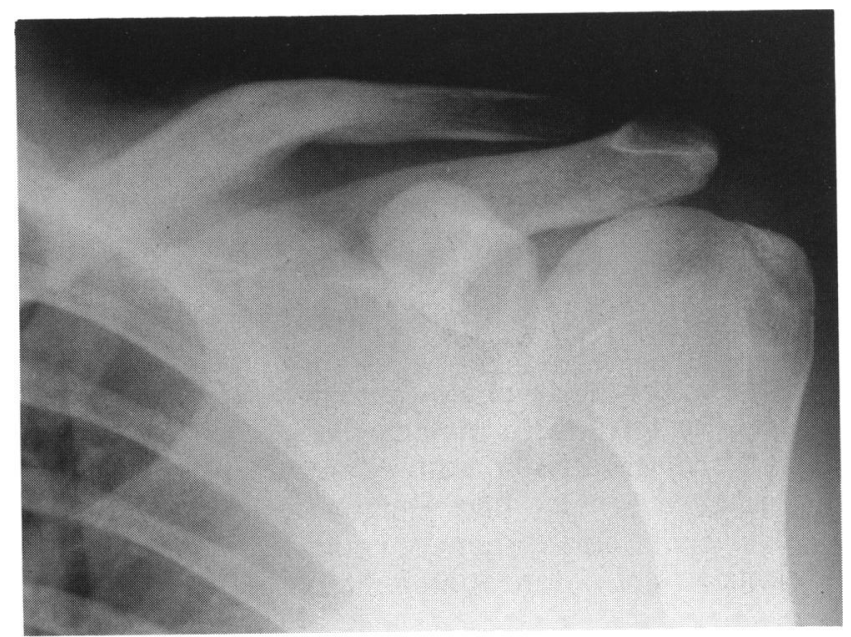

Figure 2. Case 1, second fracture - the greater tuberosity of the humerus. Note the healed clavicular fracture

\section{Case 2}

A 20-year-old wing-forward fell forward onto both outstretched hands and sustained bilateral Bennett's fractures, the left side being displaced. The left thumb was manipulated and both fractures were immobilized in plaster for 6 weeks. After 2 months' convalescence, he was able to play again. During the next season, he fell again and fractured the head of the left radius.

\section{Case 3}

A 26-year-old prop-forward fell onto his left wrist whilst running with the ball and sustained a displaced Colles' fracture which required manipulation. Two seasons later, he fractured the mid-shaft of the right radius and ulna during a tackle, which was manipulated and immobilized in plaster.

\section{Discussion}

Fracture is a relatively uncommon injury in rugby players. Due to the nature of the sport, the upper

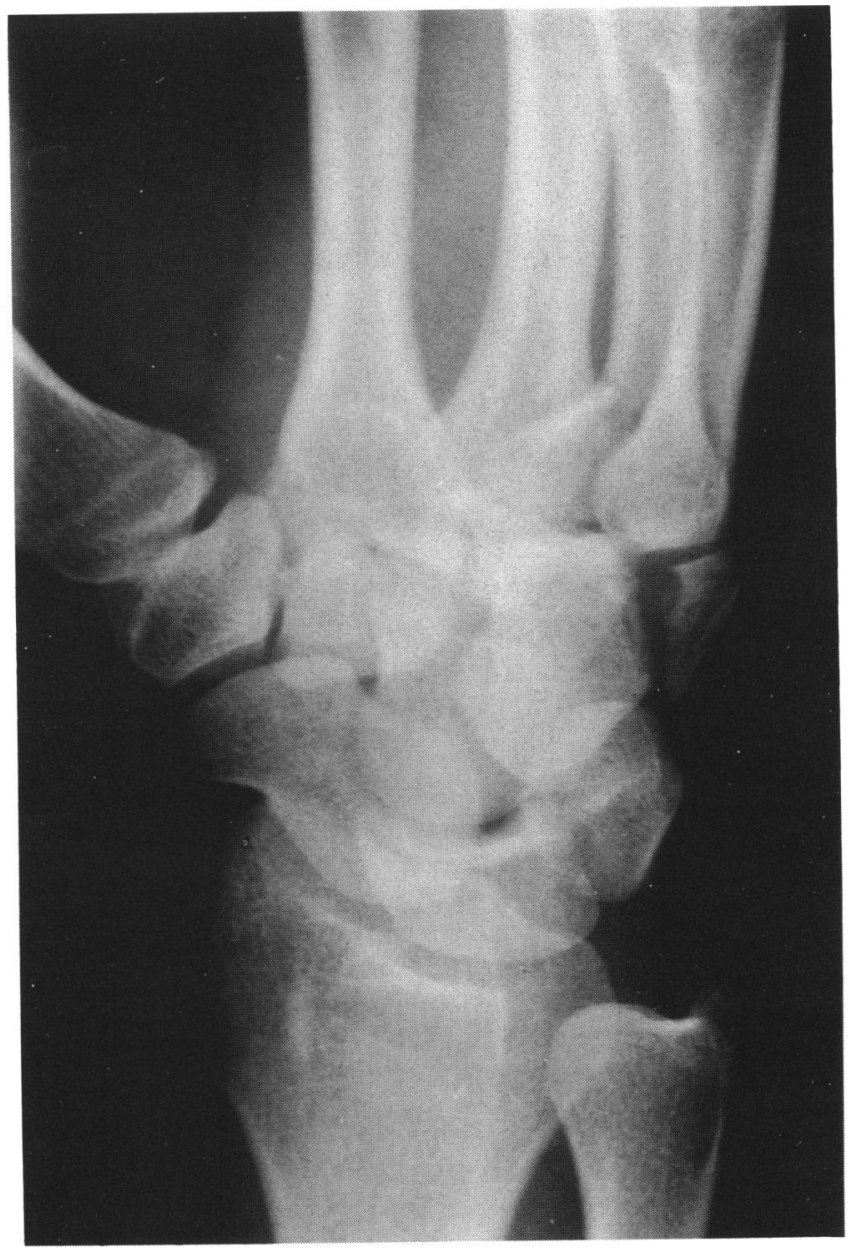

Figure 3. Case 1, third fracture - the body of the hamate and the base of the fourth metacarpal of the left hand

limb is predominantly affected, in contrast to the lower limb injuries sustained by footballers. However, data concerning rugby injuries are not well documented in the literature.

Addley and Farren reported the injuries sustained by adult rugby players in one season ${ }^{2}$. They found that $96 \%$ of the injuries (84 players in total) affected the soft tissues, $63 \%$ of the injuries affected the limbs (upper limb in $27 \%$ and lower limb in $36 \%$ ), while $4 \%$ of the injuries were fractures - of the nose, a metacarpal and a Smith's fracture. Single dislocations of the acromioclavicular joint, the temporomandibular joint and shoulder joint were reported. Interestingly, all these injuries, as in our series, affected the upper limb. It is unclear why the patients presented in our series were prone to multiple fractures since there was no evidence of underlying bone or metabolic disease. Indeed, Watters et al. stated that the vast majority of injuries occurred in fit individuals and probably arose because of general fatigue due to the pace of the game. Briscoe studied 346 sports injuries sustained by schoolboys during a 12-year period $^{1}$. By comparing the injuries sustained during football, Eton football and rugby, he showed that rugby was the most dangerous game. Whereas soft tissue injuries were more frequently observed in football players (52, 30 and $15 \%$ respectively), there was a higher incidence of minor head injuries in 
rugby players. Of the total 57 fractures, $56 \%$ affected the leg during Eton football, and only 13 and $11 \%$ occurred in the leg in football and rugby players respectively. The nose, clavicle, forearm or wrist were the sites most commonly affected. The low percentage of lower limb fractures in the schoolboy rugby players compared to the football player and the general experience of adult competitors probably reflects the lower incidence of hard tackles.

Also of interest in our series were the fractures of the hamate which have not been previously reported in rugby players.

The commonest fractures of the wrist in athletes are of the scaphoid and hook of the hamate ${ }^{4}$. Fractures of the hamate are relatively rare and account for less than $2 \%$ of all carpal fractures ${ }^{5}$. Milch classified them into hook and body fractures ${ }^{6}$, and an example of each is present in our series. Although some authors state that the incidence of both types is equal, hook fractures are probably more frequent than is appreciated, especially in sport ${ }^{7,8}$. Direct trauma or a fall on the outstretched hand, as described in our case, can lead to such an injury. Thomas and Birch ${ }^{9}$ stated that with the wrist in ulnar deviation, the proximal hamate abuts against the triquetral $^{9}$. Force is directed along the metacarpal and splits the hamate coronally. If the wrist is in radial deviation, the force splits it sagittally, as seen in our case.

\section{Conclusion}

We found, like other authors, that most injuries in rugby players occurred during tackles rather than scrummages ${ }^{10}$ and that those players in the forward positions were most prone to injury. Limb injuries account for the majority of sports injuries in rugby players, and mainly affect the soft tissues. Fractures are uncommon, but tend to affect the upper limb.

\section{Acknowledgements}

The authors wish to thank the Medical Illustration Department of the Huddersfield Royal Infirmary for their contribution to this manuscript.

\section{References}

1 Briscoe JHD. Sports injuries in adolescent boarding school boys. Br J Sports Med 1985; 19: 67-70.

2 Addley $K$, Farren J. Irish rugby injury survey: Dungannon Football Club (1986-87). Br J Sports Med 1988; 22: 22-4.

3 Watters SA, Brooks S, Elton RA, Little K. Sports injuries in an Accident and Emergency Department. Arch Emerg Med 1984; 2: 105-11.

4 Zemel NP, Stark HH. Fractures and dislocations of the carpal bones. Clin Sports Med 1986: 5: 709-24.

5 Dunn AW. Fractures and dislocations of the carpus. Surg Clin N Am 1972; 52: 1513-38.

6 Milch H. Fracture of the hamate bone. J Bone Joint Surg 1934; 16: 459-62.

7 Bowen TL. Injuries of the hamate bone. Hand 1973; 5: 235-8. 8 Bryan R, Dobyns JH. Fractures of the carpal bones other than the lunate and navicular. Clin Orthop 1980; 149: 101-11.

9 Thomas AP, Birch R. An unusual hamate fracture. Hand 1983; 15: 281-6.

10 Scottish Rugby Union. Significant Injuries in Rugby 1984-85. Edinburgh, 1985. 